\title{
Estudos de simulação CFD-DEM em um Leito de Jorro Cônico
}

\section{CFD-DEM Simulation Studies in a Conical Spouted Bed}

\author{
${ }^{1}$ Ronydes Batista Júnior; ${ }^{2}$ Kassia Graciele Santos; ${ }^{* 3}$ José Luiz Vieira Neto \\ ${ }^{1}$ Aluno do Curso de Engenharia Química, Universidade Federal do Triângulo Mineiro, Uberaba, Minas Gerais, Brasil. \\ E-mail: ronydes@hotmail.com \\ *Professor do Curso de Engenharia Química, Universidade Federal do Triângulo Mineiro, Uberaba, Minas Gerais, Brasil. \\ ${ }^{2}$ E-mail: kassiagsantos@gmail.com ${ }^{3}$ Orcid: http://orcid.org/0000-0003-0736-3974 E-mail: jose.neto@uftm.edu.br
}

RESUMO: O Método de Elementos Discretos (DEM) tem se despontado como boa alternativa para modelagem de sistemas particulados densos, já que contabiliza o efeito do tamanho e da forma real da partícula, bem como, as forças partícula-partícula e partícula-parede. Contudo, para cada material é necessário identificar os parâmetros do modelo de força de contato entre as partículas, geralmente por meio de testes como o ângulo de repouso dinâmico. Neste trabalho, foi realizado um estudo de calibração dos parâmetros do modelo de colisão Mola-Amortecedor para esferas de vidro por meio de simulações DEM. Para tal, um planejamento fatorial do tipo planejamento composto central (PCC) foi utilizado para avaliar o efeito de cada parâmetro do modelo no ângulo de repouso e encontrar quais os parâmetros afetam significativamente o ângulo simulado. Após esta calibração inicial estes parâmetros foram empregados nas simulações CFDDEM de um Leito de Jorro Cônico, equipamento este usualmente empregado em aplicações de secagem. Obteve-se uma boa concordância entre dados experimentais e simulados de velocidade e de queda de pressão de Jorro Mínimo que são parâmetros importantes no projeto deste tipo de equipamento.

Palavras-chave: Fluidodinâmica Computacional; Método de Elementos Discretos; Jorro Mínimo; Leito de Jorro.

ABSTRACT: The Discrete Element Method (DEM) has emerged as a good alternative for modeling dense particulate systems, as it reports the effect of particle size and real shape, as well as, particle-particle and particle-wall forces. However, for each material, it is necessary to calibrate the parameters of the contact force model between the particles, usually through tests such as the dynamic repose angle. In this work, a study of calibration of the parameters of the Spring-dashpot collision model of glass spheres was performed through DEM simulations. To this end, a central composite design was used to evaluate the effect of each model parameter on the dynamic repose angle and to find out which parameters significantly affect the simulated angle. After this initial calibration, these parameters were used in CFD-DEM simulations in a Conical Spouted Bed, equipment usually used in drying applications. Good agreement was obtained between the experimental data and the simulated values for the minimum spout velocity and pressure drop, which are important parameters at the design of this equipment.

Keywords: Computational Fluid Dynamics; Discrete Elements Method; Minimum Spout; Spouted Bed. 
INTRODUÇÃO

O Leito de jorro foi criado em 1954 por Mathur e Epstein, como uma variação do leito fluidizado, para secagem de trigo e usado comercialmente desde 1962 (MATHUR; EPSTEIN, 1974). Atualmente, o Leito de Jorro vem sendo empregado de forma significativa e crescente na indústria, com destaque em operações unitárias de secagem, granulação e recobrimento principalmente nos setores químico, alimentício, farmacêutico e agrícola para produtos com elevado valor agregado (EPSTEIN; GRACE, 2011).

A principal característica que torna o Leito de Jorro mais indicado para certas aplicações do que a maioria dos sistemas de contato ar-sólido convencionais é o seu padrão cíclico de movimentação de partículas, além do baixo custo e simples configuração. O movimento contínuo da fase sólida promove um alto contato fluidopartícula no interior do leito, promovendo altos coeficientes de transferência de calor e massa, bem como alta taxa de recirculação de partículas (MARQUES, 2013). Apesar da sua crescente utilização, o Leito de Jorro apresenta algumas restrições como, por exemplo: limitação da altura do leito, impossibilidade de se utilizar partículas muito finas, altas quedas de pressão para o estabelecimento do jorro e principalmente grande dificuldade para a ampliação de escala (scale-up). Dessa forma, estudos sobre a modelagem e a fluidodinâmica são essenciais para o desenvolvimento de projetos desse equipamento (LIMA, 2004).

Devido ao contínuo avanço da modelagem e simulação, a fluidodinâmica computacional tem se estabelecido como uma importante ferramenta para simulações de diversos fenômenos, permitindo propor modificações, projetar e aperfeiçoar dispositivos para várias aplicações (ROSA, 2010). Recentemente, a dinâmica dos fluidos computacional (Computacional Fluid Dynamics - CFD) tem se tornado uma ferramenta poderosa na investigação da fluidodinâmica em Leitos móveis. A principal vantagem da técnica de CFD é a possibilidade de investigar um módulo experimental através de uma ampla faixa de condições operacionais, parâmetros geométricos e materiais com diferentes propriedades (MARQUES, 2013).

Segundo Niemi (2012) existem duas abordagens básicas para a simulação de escoamentos gás-sólido densos encontrados em processos de fluidização: a abordagem Euleriana-Euleriana (método de dois fluidos, TFM) e a abordagem Euleriana-Lagrangiana (método dos Elementos Discretos, DEM). O modelo "Mola-Amortecedor" (Spring-Dashpot) trata-se de um modelo linear utilizado para a predição das forças de contato, em que o termo "mola" se refere à contribuição das forças elásticas para a resposta enquanto o termo "amortecedor" se refere à dissipação devido às deformações plásticas (SILVÉRIO, 2012). O ângulo de repouso dinâmico é formado entre a superfície interna do aglomerado de partículas e a superfície horizontal (BORTOLOTTI et al., 2013).

O objetivo geral deste trabalho é avaliar diferentes valores de parâmetros do modelo "Spring-Dashpot" para colisão entre as partículas, comparando com dados experimentais do ângulo de repouso, e estudar a interferência desses parâmetros na simulação. Posteriormente realizar simulação da movimentação dessas mesmas partículas no Leito de Jorro. Um planejamento composto central foi empregado para planejar as simulações, a fim de avaliar o efeito de cada parâmetro do modelo na resposta de ângulo de repouso dada pela simulação, de forma a encontrar quais os parâmetros afetam significativamente o ângulo simulado. Como objetivo específico deste trabalho tem-se realizar simulações numéricas acoplando a técnica de CFD com o método de Elementos Discretos, também chamadas de simulações CFD-DEM, com 
intuito de realizar simulações em um Leito de Jorro Cônico e obter parâmetros importantes de projeto, tais como, velocidade de Jorro Mínimo $\left(\mathrm{U}_{\mathrm{JM}}\right)$ e queda de pressão de jorro mínimo $\left(\Delta \mathrm{P}_{\mathrm{JM}}\right)$.

\section{MATERIAL E MÉTODOS}

\section{Material Particulado}

As partículas utilizadas neste estudo como material granular foram esferas de vidro apresentando diâmetros $\left(d_{p}\right)$ de $0,004 \mathrm{~m}$, densidade $\left(\rho_{\mathrm{p}}\right)$ de $2500 \mathrm{~kg} / \mathrm{m}^{3}$ e porosidade $\left(\varepsilon_{\mathrm{p}}\right)$ de aproximadamente 0,40 .

\section{Avaliação dos Parâmetros do Modelo de Força de Contato (Spring-Dashpot)}

O ângulo dinâmico de repouso usado neste trabalho foi obtido experimentalmente por Santos (2011) e Zhu et al. (2011) utilizando um tambor rotativo de bancada.

A fim de avaliar o efeito destes parâmetros no modelo de Elementos Discretos (DEM), sobre o ângulo dinâmico de repouso, foram realizadas simulações previstas por meio de um planejamento composto central (PCC) ortogonal com 7 réplicas no ponto central. O elevado número de réplicas são necessárias para verificar a influência dos valores de ortogonalidade $(\alpha)$.

Nesta etapa, foram calibrados no tambor os seguintes parâmetros do modelo linear "mola-amortecedor" (Spring-Dashpot), que serão usados posteriormente nas simulações de fluidodinâmica computacional acoplada ao método de elementos discretos (CFD-DEM) para o estudo no Leito de Jorro cônico: constante elástica $(k)$, coeficiente de fricção $\left(\mu_{f}\right)$ e coeficiente de restituição $(\eta)$.

Na Tabela 1 são apresentadas as condições utilizadas nas simulações do ângulo dinâmico de repouso do tambor rotativo de bancada.

Tabela 1. Condições para simulações do tambor rotativo.

\begin{tabular}{cc}
\hline Condições de Contorno & Valores \\
\hline Time step fase contínua (s) & 0,0005 \\
Time step fase discreta (s) & 0,0001 \\
Velocidade rotacional (rpm) & 25 \\
$N^{0}$ de partículas & 3229 \\
$N^{0}$ de células da malha & 445 \\
Tempo simulado (s) & 12 \\
Diâmetro do cilindro (m) & 0,05 \\
Comprimento do cilindro $(\mathrm{m})$ & 0,05 \\
\hline
\end{tabular}

Os dados experimentais empregados para validação dos resultados simulados via CFD-DEM no estudo de Leito de Jorro foram retirados dos trabalhos de Vieira Neto (2007) e Santos (2011).

O Leito de Jorro utilizado nestes trabalhos consiste de uma base cônica com diâmetro de entrada de 0,035 m e 0,15 m de altura, acoplada a uma coluna cilíndrica com $0,21 \mathrm{~m}$ de diâmetro e $0,70 \mathrm{~m}$ de altura. A base cônica do leito apresenta ângulo de inclinação de $60^{\circ}$. 


\section{RESULTADOS E DISCUSSÃO}

\section{Calibração de parâmetros do Modelo Spring-dashpot empregando simulações DEM do ângulo de repouso dinâmico}

Os resultados de ângulo dinâmico de repouso para as simulações realizadas são encontrados na Tabela 2. De acordo com experimentos realizados utilizando tambor rotativo encontrados na literatura, observa-se que o ângulo dinâmico de repouso da esfera de vidro (4 mm) é aproximadamente $32^{\circ}$ (SANTOS, 2013), sendo que em algumas condições experimentais observa-se que foram encontrados resultados próximos a este valor de ângulo.

Tabela 2. Ângulo dinâmico de repouso obtido pela simulação do PCC.

\begin{tabular}{|c|c|c|c|c|c|c|c|c|c|c|c|c|}
\hline \multirow[t]{2}{*}{ Exp. } & \multicolumn{3}{|c|}{ Variável } & \multicolumn{3}{|c|}{ Variável codificada } & \multicolumn{3}{|c|}{$\begin{array}{l}\text { Medida do ângulo } \\
\phi\left({ }^{\circ}\right) \text { em triplicata }\end{array}$} & \multirow[t]{2}{*}{ Média } & \multirow{2}{*}{$\begin{array}{l}\text { Desvio } \\
\text { padrão }\end{array}$} & \multirow{2}{*}{$\begin{array}{l}\text { Erro } \\
(\%)\end{array}$} \\
\hline & $k$ & $\mu_{f}$ & $\eta$ & $\mathrm{X}_{1}$ & $\mathrm{X}_{2}$ & $X_{3}$ & $1^{a}$ & $2^{a}$ & $3^{a}$ & & & \\
\hline 1 & 600 & 0,15 & 0,7 & -1 & -1 & -1 & 36 & 35 & 37,78 & 36,26 & 1,41 & 13,31 \\
\hline 2 & 600 & 0,15 & 0,9 & -1 & -1 & 1 & 35 & 36 & 37,09 & 36,03 & 1,05 & 12,59 \\
\hline 3 & 600 & 0,45 & 0,7 & -1 & 1 & -1 & 67 & 66 & 65,06 & 66,02 & 0,97 & 106,3 \\
\hline 4 & 600 & 0,45 & 0,9 & -1 & 1 & 1 & 58 & 57 & 58,84 & 57,95 & 0,92 & 81,08 \\
\hline 5 & 1000 & 0,15 & 0,7 & 1 & -1 & -1 & 33 & 32 & 36,19 & 33,73 & 2,19 & 5,41 \\
\hline 6 & 1000 & 0,15 & 0,9 & 1 & -1 & 1 & 34 & 33 & 33,43 & 33,48 & 0,50 & 4,61 \\
\hline 7 & 1000 & 0,45 & 0,7 & 1 & 1 & -1 & 68 & 69 & 65,06 & 67,35 & 2,05 & 110,48 \\
\hline 8 & 1000 & 0,45 & 0,9 & 1 & 1 & 1 & 60 & 58 & 62,93 & 60,31 & 2,48 & 88,47 \\
\hline 9 & 484,99 & 0,3 & 0,8 & $-1,575$ & 0 & 0 & 55 & 52 & 58,24 & 55,08 & 3,12 & 72,13 \\
\hline 10 & 1115,01 & 0,3 & 0,8 & 1,575 & 0 & 0 & 54 & 55 & 55,01 & 54,67 & 0,58 & 70,84 \\
\hline 11 & 800 & 0,064 & 0,8 & 0 & $-1,575$ & 0 & 7 & 6 & 7,52 & 6,84 & 0,77 & 78,63 \\
\hline 12 & 800 & 0,536 & 0,8 & 0 & 1,575 & 0 & 67 & 64 & 63,95 & 64,98 & 1,75 & 103,07 \\
\hline 13 & 800 & 0,3 & 0,643 & 0 & 0 & $-1,575$ & 61 & 66 & 61,93 & 62,98 & 2,66 & 96,80 \\
\hline 14 & 800 & 0,3 & 0,958 & 0 & 0 & 1,575 & 52 & 51 & 54,46 & 52,49 & 1,78 & 64,02 \\
\hline 15 & 800 & 0,3 & 0,8 & 0 & 0 & 0 & 54 & 53 & 55,71 & 54,24 & 1,37 & 69,49 \\
\hline 16 & 800 & 0,3 & 0,8 & 0 & 0 & 0 & 54 & 52 & 54,21 & 53,40 & 1,22 & 66,89 \\
\hline 17 & 800 & 0,3 & 0,8 & 0 & 0 & 0 & 54 & 55 & 55,10 & 54,70 & 0,61 & 70,94 \\
\hline 18 & 800 & 0,3 & 0,8 & 0 & 0 & 0 & 54 & 52 & 55,71 & 53,90 & 1,86 & 68,45 \\
\hline 19 & 800 & 0,3 & 0,8 & 0 & 0 & 0 & 54 & 53 & 53,57 & 53,52 & 0,50 & 67,26 \\
\hline 20 & 800 & 0,3 & 0,8 & 0 & 0 & 0 & 54 & 51 & 54,21 & 53,07 & 1,80 & 65,84 \\
\hline 21 & 800 & 0,3 & 0,8 & 0 & 0 & 0 & 54 & 56 & 53,57 & 54,52 & 1,30 & 70,39 \\
\hline
\end{tabular}

A Figura 1 apresenta os resultados encontrados pelas simulações pelo método de elementos discretos (DEM), utilizando-se o modelo mola-amortecedor (Spring-dashpot), para cada combinação de parâmetros estabelecida pelo PCC. 
As simulações 17 a 21 não estão ilustradas na Figura 1 por se tratarem de réplicas do PCC, apresentando assim valores aproximados aos obtidos pelas corridas 15 e 16 . Pelas simulações, podem-se notar alguns valores bastante discrepantes do ângulo de repouso dinâmico reportado na literatura $\left(32^{\circ}\right)$ como, por exemplo, $6,84^{\circ}$ (simulação 11 ) e $67,35^{\circ}$ (simulação 7 ), o que mostra que os parâmetros analisados afetam de maneira relevante na obtenção do ângulo de repouso. Ressalta-se pela simulação que os valores de ângulo de repouso dinâmico que mais se aproximam do experimental são os obtidos pelas simulações 5 e 6 .

Figura 1. Simulações do tambor rotativo nas condições do PCC



5

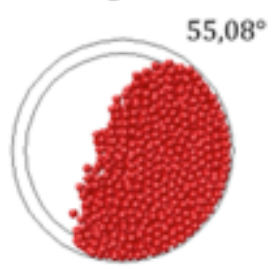

9

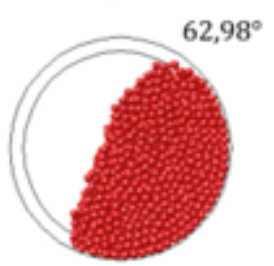

13

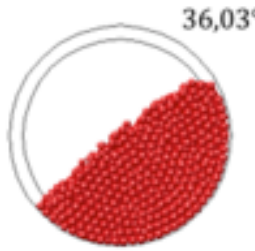

2

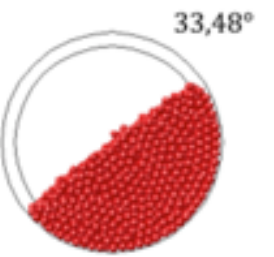

6

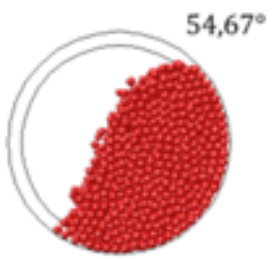

10



14

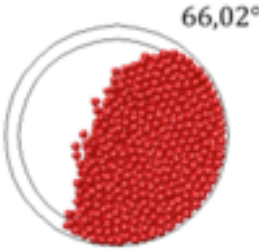

3

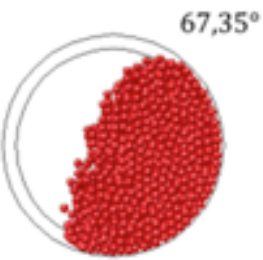

7

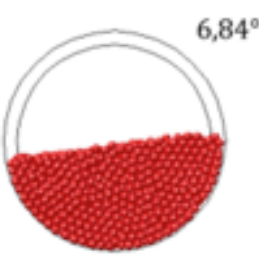

11

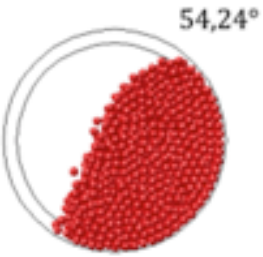

15



4

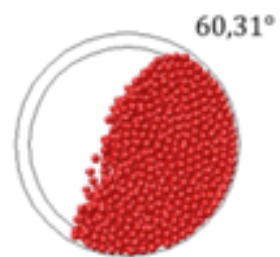

8

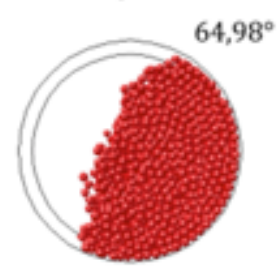

12

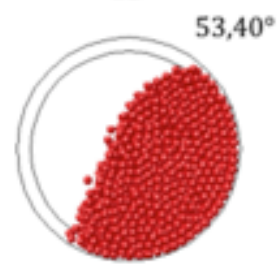

16

Por meio da análise estatística dos dados da Tabela 2, observa-se pelo valor do $\mathrm{R}^{2}$, que $97,89 \%$ dos dados utilizados são representados pelo modelo reduzido, como mostra a Equação 1.

$$
=54,18+15,639 X_{2}-7,147 X_{2}^{2}-2,401 X_{3}+1,648 X_{3}^{2}-1,954 X_{2} X_{3}
$$

De acordo com o modelo reduzido conseguido pela avaliação dos, parâmetros pode-se verificar que o ângulo de repouso dinâmico se mostrou diretamente dependente do coeficiente de fricção e inversamente dependente do coeficiente de restituição, apresentando ainda termos de efeitos quadráticos e de interação entre estas duas variáveis. 
Quanto maior a fricção, maior será a resistência da esfera de vidro ao deslizamento, enquanto que, para menores valores de restituição a velocidade após o impacto entre duas partículas é reduzida. A Figura 2 ilustra a superfície de resposta relacionando os parâmetros significativos do modelo (restituição e fricção), fixando o valor do coeficiente de elasticidade igual à zero.

Figura 2. Superfície de resposta para $k=800 \mathrm{~N} / \mathrm{m}\left(\mathrm{X}_{1}=0\right)$.

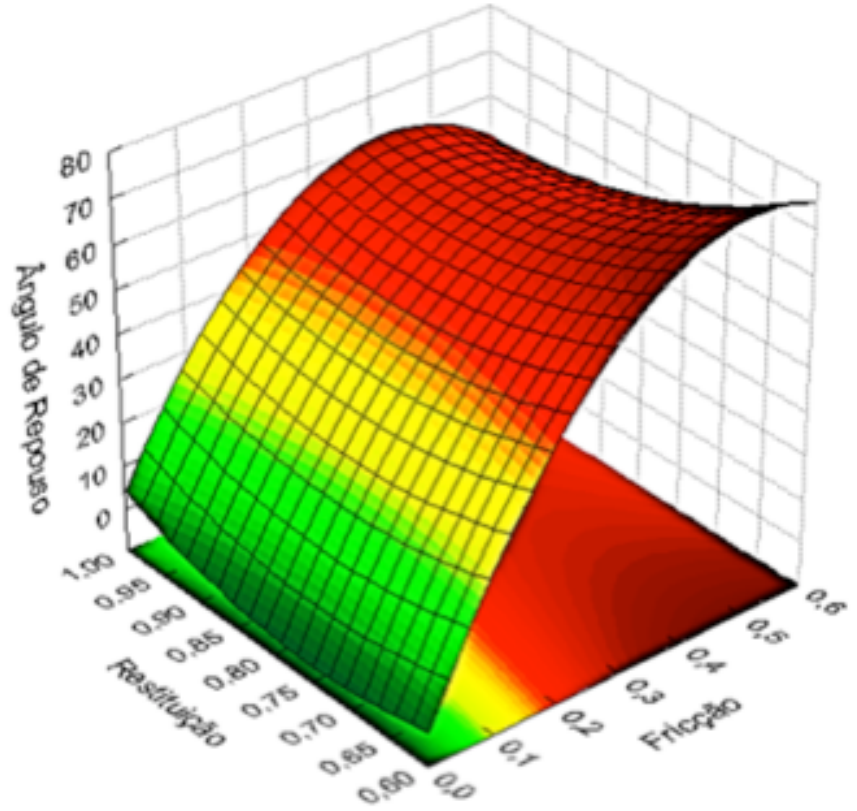

O valor desejado $\left(\phi \approx 32^{\circ}\right)$ é encontrado para maiores valores de restituição e menores valores de fricção. Como a resposta não é dependente do coeficiente de elasticidade selecionou-se um valor intermediário para o mesmo, $800 \mathrm{~N} / \mathrm{m}$, já que vários autores empregaram este valor em estudos de simulação DEM com esferas de vidro (ZHU et al., 2011). Para a determinação dos coeficientes de fricção e restituição, optou-se em selecionar a corrida 6 , pois essa apresenta o menor erro entre as simulações, portanto coeficiente de fricção é igual a 0,15 e restituição igual a 0,9.

\section{Aplicação dos Parâmetros no Leito de Jorro}

Considerando os melhores valores dos parâmetros encontrados no tópico anterior, foram realizados dois casos de simulação para aplicação em um leito de jorro, ambas utilizando: $800 \mathrm{~N} / \mathrm{m}$ para o coeficiente de elasticidade; 0,9 para o coeficiente de restituição e 0,15 para o coeficiente de fricção. Para a realização das simulações computacionais CFD-DEM, utilizaram-se as condições experimentais utilizadas por Santos (2008).

Para esferas de vidro de $1 \mathrm{~mm}$ de diâmetro, utilizou-se uma massa total de partículas igual a $346,33 \mathrm{~g}$ apresentando 203.881 partículas $\left(\mathrm{N}_{\mathrm{p}}\right)$, atingindo uma altura inicial de partículas igual a $6 \mathrm{~cm}\left(\mathrm{H}_{0}=6 \mathrm{~cm}\right)$.

A curva característica experimental de Santos (2011), Figura 3, foi utilizada para a determinação das velocidades empregadas na simulação CFD-DEM, dividindo-se a vazão encontrada no gráfico pela área de entrada do leito de jorro de $9,62 \mathrm{~cm}^{2}$. Alguns pontos 
desta curva característica foram utilizados para criar uma UDF (User Defined Function), arquivo na linguagem $C$ que gera o perfil de velocidade de escoamento do ar, que neste caso foi de $U_{J M}=1,93 \mathrm{~m} / \mathrm{s}$, que representa a velocidade de jorro mínimo.

Figura 3. Curva característica de esferas de vidro de $1 \mathrm{~mm}$ de diâmetro.



Fonte: Santos, 2011

A partir dos parâmetros obtidos pelo PCC para o modelo Spring-dashpot e determinado o perfil de velocidade pela curva característica, foi realizada a simulação CFD-DEM para o leito de jorro apresentada a seguir pela Figura 4, na velocidade de alimentação de ar no jorro mínimo de $U_{\mathrm{JM}}=1,93 \mathrm{~m} / \mathrm{s}$.

Figura 4. Simulação em leito de jorro com esferas de vidro de $1 \mathrm{~mm}$ de diâmetro, com velocidade de ar de jorro mínimo $\left(\mathrm{U}_{\mathrm{JM}}\right)$ com legenda para velocidade axial das partículas
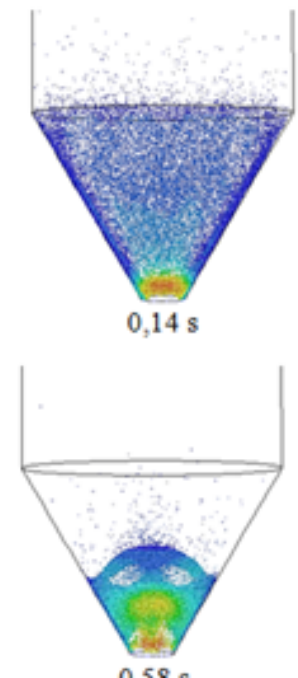

$0,58 \mathrm{~s}$
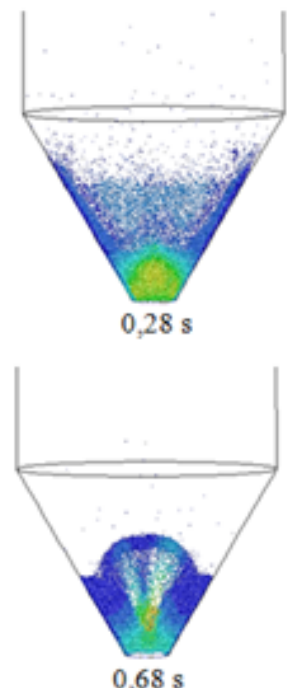

$4,1 \mathrm{~m} / \mathrm{s}$
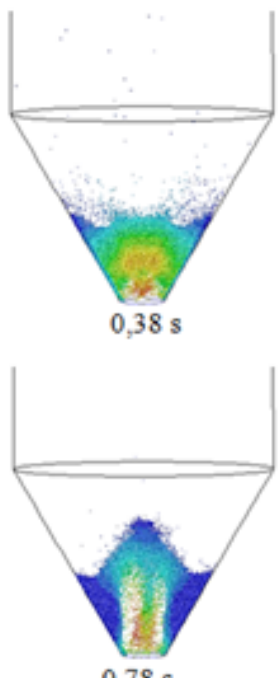

$0,78 \mathrm{~s}$
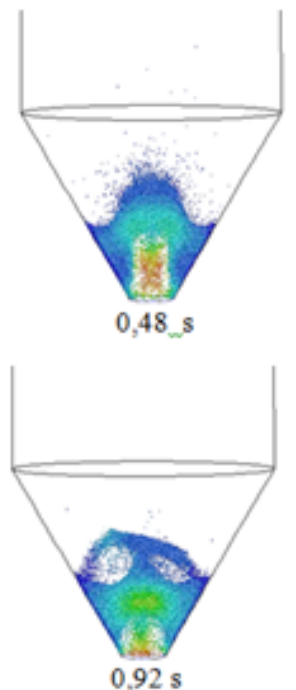

$6,9 \mathrm{~m} / \mathrm{s}$

$5,6 \mathrm{~m} / \mathrm{s}$

$2,7 \mathrm{~m} / \mathrm{s}$

$1,3 \mathrm{~m} / \mathrm{s}$

$0,0 \mathrm{~m} / \mathrm{s}$

Como o esforço computacional para esferas muito pequenas era muito grande, 
mas que verificou-se a formação da fonte e o inicio do comportamento fluidodinâmico estava de acordo com o esperado, os testes preliminares foram realizados e para viabilizar o estudo optou-se por fazer novos testes agora com esferas de vidro $4 \mathrm{~mm}$.

Assim, um segundo estudo de caso foi de simulações CFD-DEM com esferas de vidro de $4 \mathrm{~mm}$ de diâmetro, empregando uma massa total de partículas de 701,70 $\mathrm{g}$ apresentando 8.376 partículas $(\mathrm{Np})$, o que resulta em uma altura inicial de leito estático $\mathrm{H}_{0}$ $=0,08 \mathrm{~m}$.

A obtenção dos parâmetros do modelo e do perfil de velocidade ocorre de forma análoga ao caso anterior, através da curva característica experimental de Santos (2011), Figura 5, que foi utilizada para obter o perfil de velocidade de escoamento do ar, que neste caso foi de $U_{J M}=11,28 \mathrm{~m} / \mathrm{s}$, que representa a velocidade de jorro mínimo.

Figura 5. Curva característica de esferas de vidro de $4 \mathrm{~mm}$ de diâmetro.

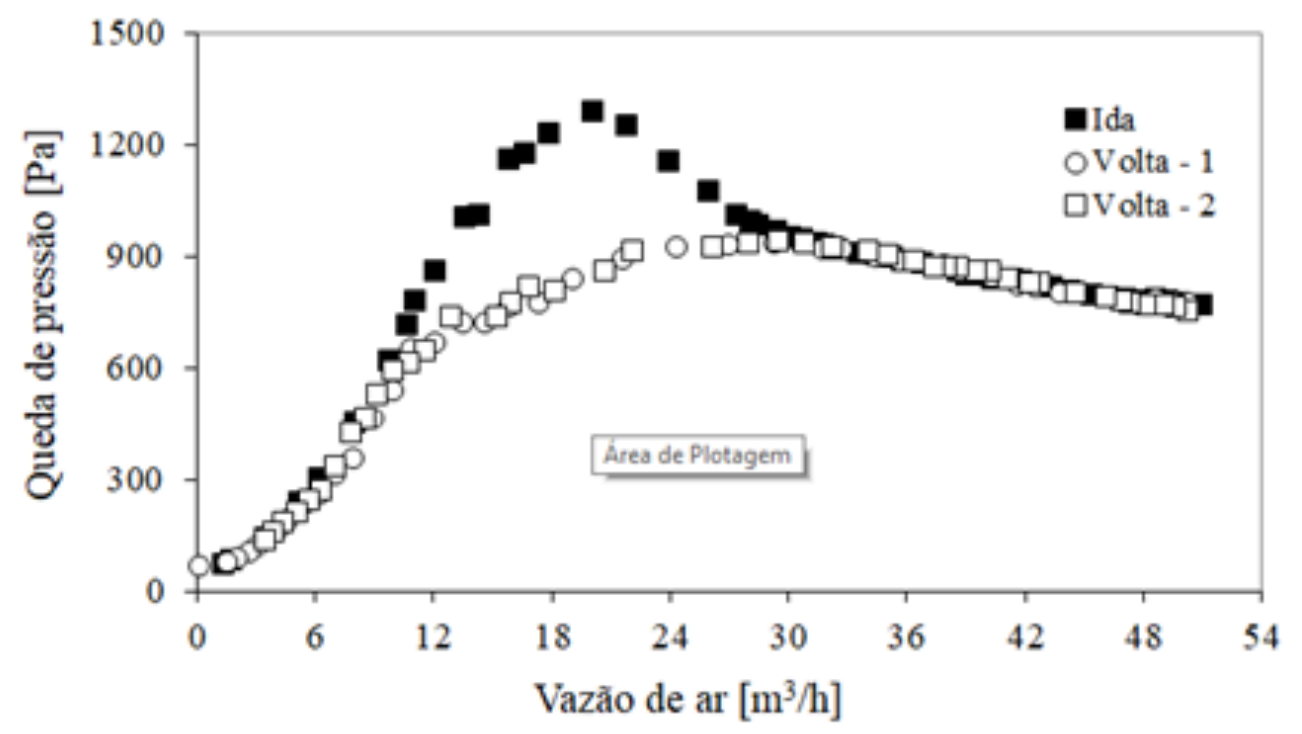

Fonte: Santos, 2011

A Figura 6 apresenta a dinâmica das partículas no leito de jorro, com legenda para velocidade de magnitude das partículas. Em baixos tempos de simulação, as partículas, que foram injetadas no leito com pequena distância uma das outras, se acomodam e a presença da alimentação de ar começa a formar uma pequena cavidade na base do leito como mostra Figura $6(a, b, c)$.

À medida que a simulação prossegue, com cerca de $1 \mathrm{~s}$ de simulação em tempo real na Figura 6 (d, e), a cavidade fica na iminência de ser rompida. Apesar da cavidade se romper e dar início a um jorro, ela quase torna a se fechar, mostrando que realmente 0 leito encontra-se na iminência de jorro, o que caracteriza a condição de jorro mínimo.

Assim, os resultados simulados mostram que a velocidade experimental de ar de jorro mínimo, $U_{\mathrm{JM}}=11,28 \mathrm{~m} / \mathrm{s}$, conduziu a simulação para uma condição física em que o leito está na iminência do jorro.

Portanto, existe uma boa concordância entre os dados experimentais de Santos (2011), com vazão de ar do jorro mínimo, $Q_{J M}=39,08 \mathrm{~m}^{3} / \mathrm{h}$, e queda de pressão do jorro mínimo, $\Delta \mathrm{P}_{\mathrm{JM}}=900 \mathrm{~Pa}$ e os valores simulados neste trabalho empregando simulações CFD-DEM. 
Figura 6. Simulação em leito de jorro com esferas de vidro de $4 \mathrm{~mm}$ de diâmetro, com velocidade de ar de jorro mínimo $\left(\mathrm{U}_{\mathrm{JM}}\right)$ com legenda para velocidade axial das partículas

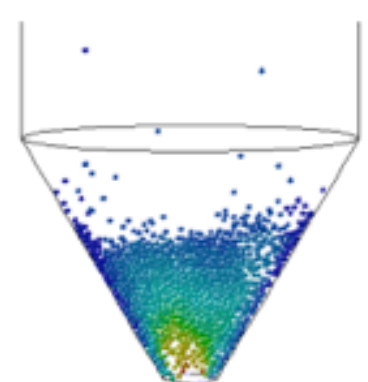

(a) $0,17 \mathrm{~s}$

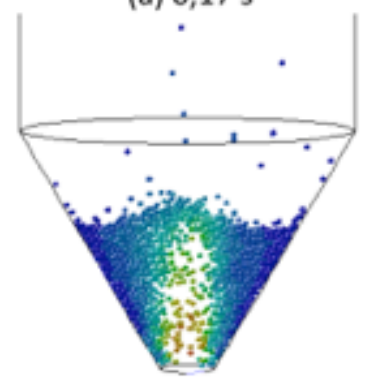

(e) $1,14 \mathrm{~s}$

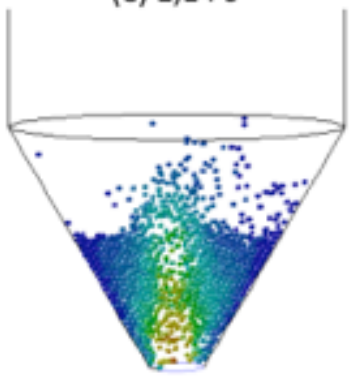

(i) $1,53 \mathrm{~s}$

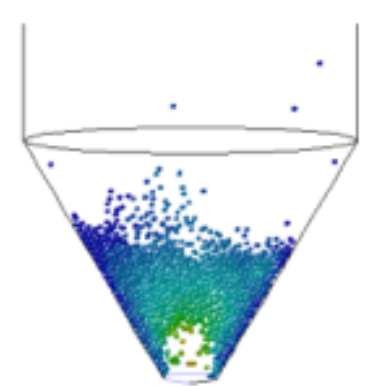

(b) $0,27 \mathrm{~s}$

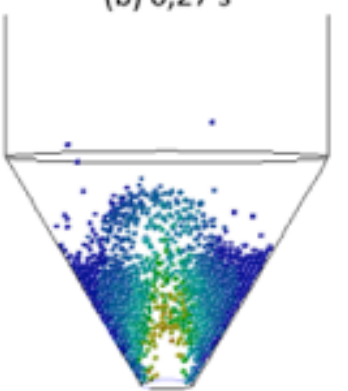

(f) $1,26 \mathrm{~s}$

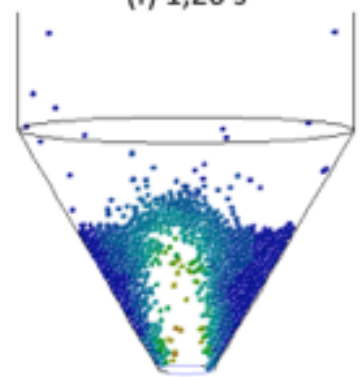

(j) $1,62 \mathrm{~s}$

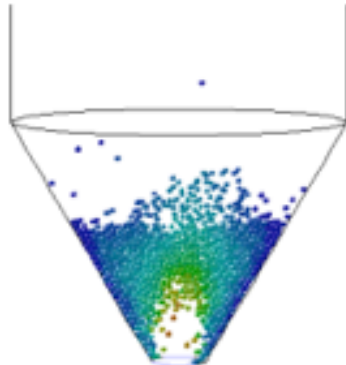

(c) $0,57 \mathrm{~s}$



(g) $1,38 \mathrm{~s}$

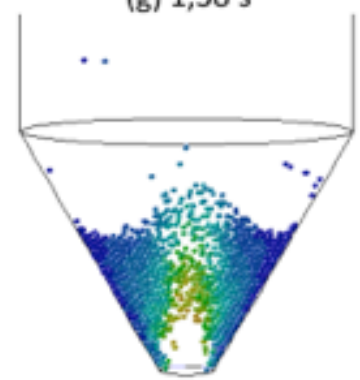

(k) $1,77 \mathrm{~s}$

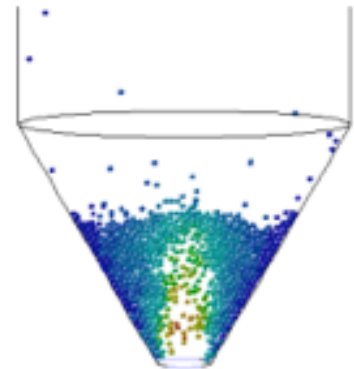

(d) $1,07 \mathrm{~s}$

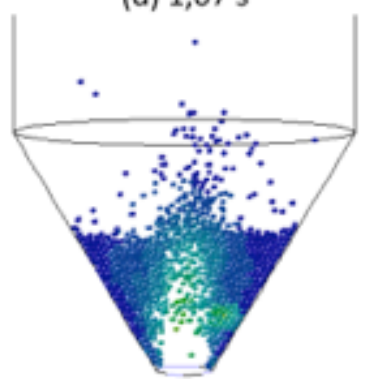

(h) $1,49 \mathrm{~s}$

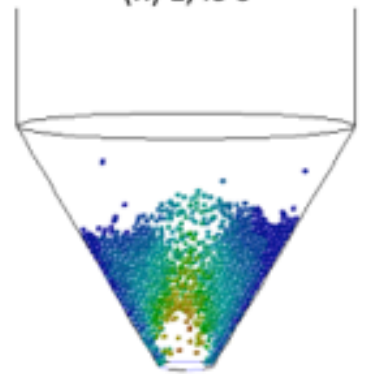

(I) $1,98 \mathrm{~s}$

\section{CONCLUSÕES}

A partir dos resultados obtidos no PCC nas simulações de calibração inicial empregando o método DEM, nota-se que o coeficiente de elasticidade não interfere no modelo mola amortecedor para a esfera de vidro. Em contrapartida, altos valores de friç̧ão e baixos valores de restituição conduzem a um aumento do ângulo de repouso dinâmico simulado das esferas de vidro.

Os estudos de casos aplicados ao Leito de Jorro apresentaram boa adequação aos parâmetros obtidos pelo PCC, especialmente para partículas maiores $(4 \mathrm{~mm})$, pois o perfil gerado pela simulação CFD-DEM corresponde ao que era esperado para condição de jorro mínimo experimental, tanto na vazão de jorro mínimo quanto na queda de pressão de jorro mínimo, e que o comportamento apresentado pelas partículas de $4 \mathrm{~mm}$ no interior do leito de jorro estão na iminência de jorro. 


\section{AGRADECIMENTOS}

Os autores agradecem o apoio financeiro provido através do Projeto FAPEMIG (APQ 01139-14 - Edital 17/2013 - PPP), ao Programa de Pós-Graduação Multicêntrico em Química (PPGMQ-MG) e a Universidade Federal do Triângulo Mineiro (UFTM).

\section{REFERÊNCIAS}

BORTOLOTTI, C.T.; SANTOS, K.G.; FRANCISQUETTI, M.C.C.; DUARTE, C.R.; BARROZO, M.A.S. Hydrodynamic study of a mixture of West Indian Cherry residue and Soybean grains in a Spouted Bed. The Canadian Journal of Chemical Engineering. v. 91, p. 1871-1880, 2013.

EPSTEIN, N.; GRACE, J.R. Spouted and Spout-Fluid Beds. New York: Cambridge University Press, 2011.

LIMA, P.M. Análise da fluidodinâmica e transferência de calor em Leito de Jorro com alimentação contínua de sólidos pela base. 2004. Dissertação (Mestrado) Universidade Federal de São Carlos, São Carlos, 2004.

MARQUES, I.I.D.R. Investigação do Leito de Jorro como Reator em potencial de Pirólise de partículas cartonadas. 2013. Dissertação (Mestrado) - Universidade Federal do Espirito Santo, São Mateus, 2013.

MATHUR, K.B.; EPSTEIN, N. Spouted Beds. New York: Academic Press, 1974.

NIEMI, T. Particle size distribution in CFD simulation of gas-particle flows. 2012. M.Sc. thesis, Aalto University, Finland, 2012.

ROSA, C.A. Estudo experimental e numérico da fluidodinâmica e das transferências de calor e massa em um Leito de Jorro Contínuo: Uso da Técnica CFD. 2010. Tese de Doutorado, Universidade Federal de São Carlos, São Carlos, 2010.

SANTOS, K.G. Aspectos fundamentais da Pirólise de Biomassa em Leito de Jorro: Fluidodinâmica e cinética do processo. 2011. Tese (Doutorado) - Universidade Federal de Uberlândia, Uberlândia, 2011.

SANTOS, K.G. Estudo da fluidodinâmica do Leito de Jorro por CFD com malhas tridimensionais. 2008. Dissertação (Mestrado). - Universidade Federal de Uberlândia, Uberlândia, 2008.

SILVÉRIO, B.C. Estudos fluidodinâmicos e de secagem de fertilizantes em diferentes tipos de Secadores Rotatórios. 2012. Tese (Doutorado) - Universidade Federal de Uberlândia, Uberlândia, 2012. 
VIEIRA NETO, J.L. Estudo experimental e de simulação da fluidodinâmica de um Leito de Jorro com Tubo Draft. 2007. Dissertação (Mestrado). - Universidade Federal de Uberlândia, Uberlândia, 2007.

ZHU, R.R.; ZHU, W.B.; XING, L.C.; SUN Q.Q. DEM simulation on particle mixing in dry and wet particles spouted bed. Powder Technology. v. 210, p. 73-81, 2011.

Recebido em: 29/06/2019

Aprovado em: 23/08/2019 